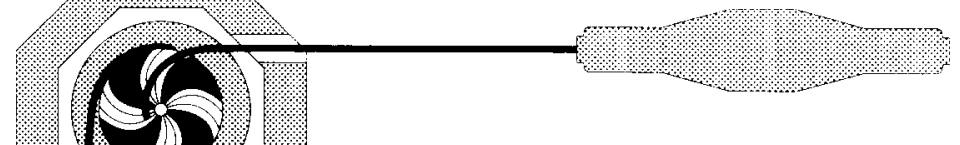

\title{
Evidence for octupole correlations at high spins in neutron-deficient ${ }^{110} \mathrm{Te}$
}

\author{
E.S. Paul ${ }^{1}$, H.R. Andrews ${ }^{2}$, T.E. Drake ${ }^{3}$, J. DeGraaf', V.P. Janzen ${ }^{2}$, S. Pilotte ${ }^{4}$, \\ D.C. Radford ${ }^{2}$, and D. Ward ${ }^{2}$
}

'Oliver Lodge Laboratory, University of Liverpool, PO Box 147, Liverpool L69 3BX, UK $j w 943$

${ }^{2}$ Chalk River Laboratories, AECL Research, Chalk River, Ontario, KOJ 1J0, Canada

${ }^{3}$ Department of Physics, University of Toronto, Toronto, Ontario, M5S 1A7, Canada

${ }^{4}$ Department of Physics, University of Ottawa, Ottawa, Ontario, KIN 6N5, Canada

Published in Phys. Rev. C50, Rapid Comm. 534

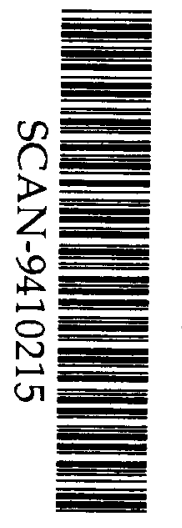

\section{$\underline{\text { NOTICE }}$}

This report is not a formal publication; if it is cited as a reference, the citation should indicate that the report is unpublished. To request copies our E-Mail address is TASCC@CRL.AECL.CA.

Physical and Environmental Sciences

Chalk River Laboratories

Chalk River, ON KOJ 1 JO Canada

1994 September 


\title{
Evidence for octupole correlations at high spins in neutron-deficient ${ }^{110} \mathrm{Te}$
}

\author{
E.S. Paul ${ }^{1}$, H.R. Andrews ${ }^{2}$, T.E. Drake ${ }^{3}$, J. DeGraaf ${ }^{3}$, V.P. Janzen ${ }^{2}$, S. Pilotte ${ }^{4}$, \\ D.C. Radford ${ }^{2}$, and D. Ward ${ }^{2}$ \\ 1 Oliver Lodge Laboratory, University of Liverpool, PO Box 147, Liverpool L69 3BX, UK \\ ${ }^{2}$ Chalk River Laboratories, AECL Research, Chalk River, ON, KOJ 1JO, Canada \\ ${ }^{3}$ Department of Physics, University of Toronto, Toronto, Ontario M5S 1A7, Canada \\ ${ }^{4}$ Department of Physics, University of Ottawa, Ottawa K1N 6N5, Canada
}

(April 30, 1994)

\begin{abstract}
States in neutron-deficient ${ }^{110} \mathrm{Te}$ have been studied with the ${ }^{58} \mathrm{Ni}+{ }^{58} \mathrm{Ni}$ reaction at $250 \mathrm{MeV}$. An unusual feature is that, above spin $8^{+}$, negative parity states become yrast. These states are interpreted in terms of a $\nu\left[h_{11 / 2} \otimes d_{5 / 2}\right]$ structure $(\Delta l=\Delta j=3)$. For spins above $15^{-}$, strong $(E 1)$ dipoles are seen linking another band into the negative-parity states. This is taken as evidence for octupole correlations in ${ }^{110} \mathrm{Te}$ at high spin.
\end{abstract}

NUCLEAR REACTIONS ${ }^{58} \mathrm{Ni}+{ }^{58} \mathrm{Ni}$ at $250 \mathrm{MeV}$, enriched targets, HPGe detectors, BGO suppression shields, BGO multiplicity filter, measured $\mathrm{E}_{\gamma}, I_{\gamma \gamma}(\theta)$, deduced $I^{\pi}$.

PACS numbers: 21.10.Re, 27.70+q, 23.20.Lv.

Typeset using REVTEX 
The neutron-deficient $(N \sim 60)$ tellurium $(Z=52)$ and xenon $(Z=54)$ isotopes are interesting because they lie in one of a very few regions of the nuclear chart where orbitals differing in total and orbital angular momentum by $3 \hbar$ approach the Fermi surface for both protons and neutron. The interaction of such $\Delta j=\Delta l=3$ orbitals is expected to lead to octupole correlations; the onset of octupole shapes has indeed been predicted for $\mathrm{N} \leq 58$ [1]. In this study of ${ }^{110} \mathrm{Te}(\mathrm{N}=58)$, we find a sequence of interleaved states of negative and positive parity at high spins which are connected by very strong E1 transitions; this is good evidence for octupole correlations. Similar results have been seen in ${ }^{114} \mathrm{Xe}(\mathrm{N}=60)$ [2], albeit at lower spins, but the enhancement of the E1 transitions in this case appears to be small. The ${ }^{114} \mathrm{Xe}$ results have been taken to be an indication that the E1 rates are reduced at small isospin, $\mathrm{T}_{Z}$; our new results for ${ }^{110} \mathrm{Te}$, however, imply that if such a scaling exists, it is much smaller than suggested in Ref. [2].

High-spin states in neutron-deficient ${ }^{110} \mathrm{Te}$ were populated with the ${ }^{58} \mathrm{Ni}\left({ }^{58} \mathrm{Ni}, \alpha 2 \mathrm{p}\right){ }^{110} \mathrm{Te}$ reaction at $250 \mathrm{MeV}$. The ${ }^{58} \mathrm{Ni}$ beam, provided by the Tandem Accelerator Superconducting Cyclotron (TASCC) facility at the Chalk River Laboratories of AECL Research, was incident upon a $1 \mathrm{mg} / \mathrm{cm}^{2}{ }^{58} \mathrm{Ni}$ target with a thick ${ }^{197} \mathrm{Au}$ backing. Coincident $\gamma-\gamma$ data were acquired with the $8 \pi$ spectrometer, which consists of 20 Compton-suppressed HPGe detectors plus a 71-element bismuth germanate (BGO) inner ball calorimeter which provides $\gamma$-ray sum energy $H$ and fold $K$ information. Data were written onto magnetic tape for events in which two or more suppressed HPGe detectors registered in prompt time coincidence with ten or more elements of the inner ball (fold $K \geq 10$ ). Approximately $3.5 \times 10^{7}$ events were recorded to tape.

In the off-line analysis for ${ }^{110} \mathrm{Te}(\alpha 2 \mathrm{p}$ channel $)$, only events with a total sum-energy $H \geq 13$ $\mathrm{MeV}$ were incremented into a symmetrised $E_{\gamma}-E_{\gamma}$ matrix; this condition greatly suppressed events from the competing 4- and 5-particle evaporation channels, in particular ${ }^{112} \mathrm{Te}(4 \mathrm{p})$. 
We obtained angular-correlation information from the coincidence data by sorting subsets of the data recorded by HPGe detectors at specific angles with respect to the beam axis. A matrix was constructed with data from detectors at $\pm 37^{\circ}$ on one axis and detectors at $\pm 79^{\circ}$ on the second axis. Angular intensity ratios $I_{\gamma}\left(37^{\circ}, 79^{\circ}\right) / I_{\gamma}\left(79^{\circ}, 37^{\circ}\right)$ could readily be extracted from this matrix by gating on stretched quadrupole transitions. These intensity ratios were used to assist in the assignment of transition multipolarities by the method of directional correlation from oriented states (DCO) [3]. For the present geometry, intensity ratios of 1.0 are predicted for a stretched-quadrupole $\leftrightarrow$ stretched-quadrupole correlation and $\approx 0.60$ for a stretched-quadrupole $\leftrightarrow$ stretched-dipole (no mixing) correlation [4]. The values for mixed M1/E2 transitions are perturbed from this value depending on the sign and magnitude of the multipole mixing ratio $\delta$.

The nucleus ${ }^{110} \mathrm{Te}$ is the lightest even Te isotope with known $\gamma$-ray transitions. The $2^{+} \rightarrow 0^{+}$transition in ${ }^{110} \mathrm{Te}(658 \mathrm{keV})$ was first identified following the $\beta$ decay of ${ }^{114} \mathrm{Cs}$ [5], i.e. ${ }^{114} \mathrm{Cs} \stackrel{\beta}{\longrightarrow}{ }^{114} \mathrm{Xe} \stackrel{\alpha}{\longrightarrow}{ }^{110} \mathrm{Te}$; this assignment has been confirmed from mass-gated $\gamma$-ray spectra recently obtained with the Eurogam array at Daresbury, UK [6]. The level scheme deduced for ${ }^{110} \mathrm{Te}$ from the present work is presented in Fig. 1; the analysis was greatly facilitated by the use of the ESCL8R code [7]. Examples of gated coincidence spectra are shown in Fig. 2, while Table I lists energies, intensities and DCO results.

The strong low-spin transitions in ${ }^{110} \mathrm{Te}$ could be followed up to $I^{\pi}=8^{+}$. Several other lowspin positive-parity states were established from the DCO analysis. Above these positiveparity states, a strongly populated negative-parity sequence is observed. DCO ratios for the 448- and 390- keV transitions depopulating the assigned $9^{-}$band-head are consistent with pure stretched dipole transitions (E1). A second sequence of negative-parity states, shown to the left in Fig. 1, was established built on an $8^{-}$state. At higher spin, a sequence was established that decays into the negative-parity states via several dipole transitions. The 
DCO ratios obtained for the linking $1036,768,593$, and $467 \mathrm{keV}$ transitions $(\sim 0.6)$ suggest pure dipole (E1) transitions; hence positive parity is assigned to this high-spin sequence. Another band (764-945-1009) feeds into the yrast states at $I^{\pi}=15^{-}$via the $795 \mathrm{keV}$ dipole transition. The DCO ratio obtained for this linking transition $(0.56 \pm 0.07)$ is close to the value expected for a pure $\Delta I=1$ dipole transition, hence $I^{\pi}=16^{+}$may tentatively be assigned to the bandhead.

Similar to heavier even Te isotopes, the ground-state band in ${ }^{110} \mathrm{Te}$ has a vibrational rather than rotational appearance; the $E\left(4^{+}\right) / E\left(2^{+}\right)$energy ratio of 2.13 is much closer to the pure vibrational limit (2.00) than the rotational limit (3.33). Systematics of the groundstate bands in the light Te isotopes [8] show that the $2^{+}$and $4^{+}$states lie lowest in ${ }^{120} \mathrm{Te}$ $(\mathrm{N}=68)$. In lighter isotopes, these states increase in excitation energy until ${ }^{114} \mathrm{Te}(\mathrm{N}=62)$ is reached. For the very lightest isotopes, ${ }^{112} \mathrm{Te}$ and ${ }^{110} \mathrm{Te}$, the $2^{+}$and $4^{+}$states decrease in energy. The energies of the $6^{+}$and $8^{+}$states reach a maximum for ${ }^{112} \mathrm{Te}$ before decreasing in ${ }^{110} \mathrm{Te}$.

Several positive-parity states are shown to the left and right of the ground-state band in Fig. 1. The former levels consist of even-spin and odd-spin members connected by both dipole and quadrupole transitions. The low-lying states with $I^{\pi} \leq 6^{+}$in even Te isotopes $(Z=52)$ have been interpreted in terms of phonon states where $\pi\left[g_{7 / 2}\right]^{2}$ or $\pi\left[g_{7 / 2} d_{5 / 2}\right]$ proton configurations are coupled to the spherical Sn $(Z=50)$ core states $[8,9]$. The even-spin positive-parity levels shown to the right may form part of the $\gamma$-vibrational band in ${ }^{110} \mathrm{Te}$. These states show the characteristic decay for such a band into the ground-state band, namely both $\Delta I=2$ and $\Delta I=0$ transitions are observed.

The change of parity along the yrast line in ${ }^{110} \mathrm{Te}$, observed via the $448 \mathrm{keV} 9^{-} \rightarrow 8^{+}$ transition, is unique in the light even Te isotopes. In the heavier isotopes, the yrast levels continue as positive-parity states beyond low lying $10^{+}$states and may be interpreted 
in terms of an aligned $\nu\left[h_{11 / 2}\right]^{2}$ structure. For ${ }^{110} \mathrm{Te}$, however, the neutron Fermi surface lies below the $h_{11 / 2}$ subshell making the $\nu\left[h_{11 / 2}\right]^{2}$ configuration energetically unfavourable (the same argument is true for the $\pi\left[h_{11 / 2}\right]^{2}$ configuration), thus allowing negative-parity configurations to compete. By inspection of a single-particle diagram relevant for ${ }^{110} \mathrm{Te}$, the low-lying negative-parity sequences can be interpreted as based on the two-quasineutron $\nu\left[h_{11 / 2} \otimes d_{5 / 2}\right]$ structure. Furthermore, the high spin positive-parity states with $I^{\pi} \geq 16^{+}$ can be interpreted in terms of negative-parity $\left[h_{11 / 2} \otimes d_{5 / 2}\right]$ structures for both neutrons and protons, yielding positive parity overall. Since these orbitals differ in both $l$ and $j$ by 3 , octupole correlations may be important in this nucleus at high spin. Indeed, octupole softness at low spin has been predicted in this neutron-deficient mass region [1] and experimental evidence has recently been published for the nucleus ${ }^{114} \mathrm{Xe}[2]$.

We have extracted values for the magnitude of the intrinsic dipole moment in ${ }^{110} \mathrm{Te}$ by measuring $\mathrm{B}(\mathrm{E} 1 ; I \rightarrow I-1) / \mathrm{B}(\mathrm{E} 2 ; I \rightarrow I-2)$ ratios at high spin $(I \geq 18)$. Values of $\mathrm{B}(\mathrm{E} 1) / \mathrm{B}(\mathrm{E} 2) \sim 10^{-6} \mathrm{fm}^{-2}$ were found, as detailed in Table 2. The dipole moment was extracted with the prescription given in Ref. [10]. A quadrupole moment of $Q_{0}=200$ e.fm ${ }^{2}$ was used, which is based on a predicted quadrupole deformation of $\beta_{2} \sim 0.15$ obtained from cranking calculations using the total routhian surface (TRS) formalism [11]. The values obtained for the intrinsic dipole moment in ${ }^{110} \mathrm{Te}$ are similar to those found in the neutron-rich barium $(Z=56)$ nuclei and are slightly smaller than values typical of the Ra-Th region (see [10] and references therein); they are, however, much larger than those found in ${ }^{114} \mathrm{Xe}[2]$. The nuclei ${ }^{110} \mathrm{Te}$ and ${ }^{114} \mathrm{Xe}$ both have $\mathrm{T}_{Z}=3$, whereas the other known regions of octupole correlations occur in nuclei with $\mathrm{T}_{Z} \sim 16$ (neutron-rich $\mathrm{Ba}$ region) and $\mathrm{T}_{Z} \sim 20$ (Ra-Th region). The quantity ( $\mathrm{N}-\mathrm{Z})^{2} / \mathrm{A}^{2}$ was also discussed in Ref. [2] as a scaling parameter for the dipole moment, but we note that this parameter is nearly the same in ${ }^{110} \mathrm{Te}$ and ${ }^{114} \mathrm{Xe}$.

To summarize, high spin states have been identified in ${ }^{110} \mathrm{Te}$ for the first time. Between 
spins 8 and 17 , negative-parity states, based on the $\nu\left[h_{11 / 2} \otimes d_{5 / 2}\right]$ configuration, become yrast. At higher spin, a positive-parity structure is connected to the negative-parity states by strong dipole $(E 1)$ transitions. This is interpreted in terms of octupole correlations and a value for the intrinsic dipole moment has been deduced from measured $B(E 1) / B(E 2)$ ratios.

We conclude that the $\mathrm{B}(\mathrm{E} 1)$ strengths, or dipole moments in ${ }^{110} \mathrm{Te}$ are comparable to those seen in the neutron-rich barium region, and are about an order of magnitude larger than found in ${ }^{114} \mathrm{Xe}$. This reopens the question as to how the dipole moment scales with $\mathrm{T}_{Z}$, which on the basis of our work would appear to be a much weaker dependence than claimed in Ref. [2].

This work was in part supported by AECL Research, and by grants from the Natural Sciences and Engineering Research Council of Canada, and the UK SERC. 


\section{REFERENCES}

[1] J. Skalski, Phys. Lett. B238, 6 (1990).

[2] S.L. Rugari et al., Phys. Rev. C 48, 2078 (1993).

[3] K.S. Krane, R.M. Steffen, and R.M. Wheeler, Nuclear Data Tables A11, 351 (1973).

[4] D. Ward et al., Nucl. Phys. A529, 315 (1991).

[5] P. Tidemand-Petersson et al., Nucl. Phys. A437, 342 (1985).

[6] E.S. Paul et al., unpublished.

[7] D.C. Radford, in Proceedings of the International Seminar on The Frontier of Nuclear Spectroscopy, October, 1992, Kyoto, edited by Y. Yoshizawa, H. Kusakari and T. Otsuka (World Scientific, 1993, Singapore) p.229.

[8] T. Lönnroth, A. Virtanen, and J. Hattula, Physica Scripta 34, 682 (1986).

[9] P. Chowdhury, W.F. Piel, Jr., and D.B. Fossan, Phys. Rev. C 25, 813 (1982).

[10] P.A. Butler and W. Nazarewicz, Nucl. Phys. A533, 249 (1991).

[11] R. Wyss, J. Nyberg, A. Johnson, R. Bengtsson, and W. Nazarewicz, Phys. Lett. B215, $211(1988)$. 


\section{TABLES}

Table I. Gamma-ray energies, intensities, and angular correlation data for transitions assigned to ${ }^{110} \mathrm{Te}$.

\begin{tabular}{ccclr}
\hline $\mathrm{E}_{\gamma}(\mathrm{keV})^{a} \mathrm{I}_{\gamma}(\%)^{b}$ & ${\frac{I_{\gamma}\left(37^{\circ}, 79^{\circ}\right)}{I_{\gamma}\left(79^{\circ}, 37^{\circ}\right)}}^{c}$ Mult. & Assignment \\
\hline \hline 214.6 & 1.5 & $1.16(27)$ & $\mathrm{M} 1 / \mathrm{E} 2$ & $6^{+} \rightarrow 6^{+}$ \\
228.6 & 3.8 & $1.26(14)$ & $\mathrm{E} 2$ & $9^{-} \rightarrow 7^{-}$ \\
253.0 & 1.0 & $0.79(14)$ & $\mathrm{M} 1 / \mathrm{E} 2$ & $8^{+} \rightarrow 7^{+}$ \\
293.6 & 7.9 & $0.90(06)$ & $\mathrm{M} 1 / \mathrm{E} 2$ & $6^{+} \rightarrow 6^{+}$ \\
328.5 & 5.7 & $1.35(12)$ & $\mathrm{M} 1 / \mathrm{E} 2$ & $6^{+} \rightarrow 5^{+}$ \\
389.7 & 19.2 & $0.59(03)$ & $\mathrm{E} 1$ & $9^{-} \rightarrow 8^{+}$ \\
447.9 & 39.9 & $0.62(02)$ & $\mathrm{E} 1$ & $9^{-} \rightarrow 8^{+}$ \\
467.2 & 2.0 & $0.57(08)$ & $\mathrm{E} 1$ & $22^{+} \rightarrow 21^{-}$ \\
513.4 & 6.4 & $0.73(10)$ & $\mathrm{M} 1 / \mathrm{E} 2$ & $4^{+} \rightarrow 4^{+}$ \\
519.7 & 6.3 & $0.63(05)$ & $\mathrm{E} 1$ & $8^{-} \rightarrow 7^{+}$ \\
524.1 & 6.4 & $1.02(07)^{d}$ & $(\mathrm{E} 1)$ & $8^{-} \rightarrow 8^{+}$ \\
525.5 & 9.3 & $1.02(07)^{d}$ & $(\mathrm{E} 2)$ & $6^{+} \rightarrow 4^{+}$ \\
553.3 & 10.6 & $1.00(07)$ & $\mathrm{E} 2$ & $10^{-} \rightarrow 8^{-}$ \\
573.9 & 8.0 & $0.28(04)$ & $\mathrm{M} 1 / \mathrm{E} 2$ & $7^{+} \rightarrow 6^{+}$ \\
593.2 & 7.8 & $0.66(07)$ & $\mathrm{E} 1$ & $20^{+} \rightarrow 19^{-}$ \\
614.0 & 2.8 & - & & $5^{+} \rightarrow$ \\
618.3 & 72.3 & $1.00(03)$ & $\mathrm{E} 2$ & $11^{-} \rightarrow 9^{-}$ \\
647.3 & 10.5 & $1.16(07)^{d}$ & $(\mathrm{E} 1)$ & $9^{-} \rightarrow 8^{+}$ \\
648.6 & 13.5 & $1.16(07)^{d}$ & $(\mathrm{E} 2)$ & $8^{+} \rightarrow 6^{+}$ \\
657.7 & $\equiv 100$ & $1.00(04)$ & $\mathrm{E} 2$ & $2^{+} \rightarrow 0^{+}$ \\
\hline & & & &
\end{tabular}


Table I. Continued

\begin{tabular}{|c|c|c|c|c|}
\hline $\mathrm{E}_{\gamma}(\mathrm{keV})^{\circ}$ & $\mathrm{I}_{\gamma}(\%)^{b}$ & $\frac{I_{\gamma}\left(37^{\circ}, 79^{\circ}\right)}{I_{\gamma}\left(79^{\circ}, 37^{\circ}\right)}$ & ${ }^{c}$ Mult. & Assignment \\
\hline 671.9 & 11.7 & $1.05(07)$ & $\mathrm{E} 2$ & $12^{-} \rightarrow 10^{-}$ \\
\hline 722.9 & 10.5 & $1.10(08)$ & $\mathrm{E} 2$ & $14^{-} \rightarrow 12^{-}$ \\
\hline 725.6 & 6.6 & - & (E2) & $18^{+} \rightarrow 16^{+}$ \\
\hline 728.3 & 65.0 & $1.01(03)$ & $\mathrm{E} 2$ & $13^{-} \rightarrow 11^{-}$ \\
\hline 744.9 & 95.1 & $0.99(03)$ & $\mathrm{E} 2$ & $4^{+} \rightarrow 2^{+}$ \\
\hline 763.8 & 8.2 & $0.99(12)$ & $\mathrm{E} 2$ & $18 \rightarrow 16$ \\
\hline 767.6 & 12.1 & $0.58(06)$ & E1 & $18^{+} \rightarrow 17^{-}$ \\
\hline 773.1 & 4.7 & - & & $\rightarrow 15^{-}$ \\
\hline 786.5 & 57.7 & $0.99(04)$ & E2 & $15^{-} \rightarrow 13^{-}$ \\
\hline 789.8 & 7.6 & $0.50(08)$ & $\mathrm{M} 1 / \mathrm{E} 2$ & $5^{+} \rightarrow 4^{+}$ \\
\hline 795.3 & 7.0 & $0.56(07)$ & Dipole & $16 \rightarrow 15^{-}$ \\
\hline 813.4 & 10.9 & $0.89(12)$ & (E2) & $20^{+} \rightarrow 18^{+}$ \\
\hline 824.7 & 64.7 & $0.98(07)$ & $\mathrm{E} 2$ & $6^{+} \rightarrow 4^{+}$ \\
\hline 827.0 & 23.1 & $0.91(16)$ & $\mathrm{E} 2$ & $8^{+} \rightarrow 6^{+}$ \\
\hline 863.0 & 6.9 & $1.35(17)$ & $\mathrm{E} 2$ & $8^{+} \rightarrow 6^{+}$ \\
\hline 899.5 & 2.5 & - & & $\rightarrow 22^{+}$ \\
\hline 921.3 & 1.1 & 一 & & $\rightarrow 2^{+}$ \\
\hline 924.6 & 1.3 & - & & \\
\hline 944.3 & 1.5 & $1.18(16)^{d}$ & (E2) & \\
\hline 945.8 & 3.4 & $1.18(16)^{d}$ & (E2) & $18 \rightarrow 16$ \\
\hline 971.8 & 9.7 & $0.96(11)$ & $\mathrm{E} 2$ & $22^{+} \rightarrow 20^{+}$ \\
\hline 984.8 & 6.1 & $0.93(12)$ & $\mathrm{E} 2$ & $16^{-} \rightarrow 14^{-}$ \\
\hline
\end{tabular}


Table I. Continued

\begin{tabular}{rrrrr}
\hline $\mathrm{E}_{\gamma}(\mathrm{keV})^{a} \mathrm{I}_{\gamma}(\%)^{b} \frac{I_{\gamma}\left(37^{\circ}, 79^{\circ}\right)}{I_{\gamma}\left(79^{\circ}, 37^{\circ}\right)}{ }^{c}$ Mult. Assignment \\
\hline \hline 987.9 & 14.0 & $1.08(09)$ & $\mathrm{E} 2$ & $19^{-} \rightarrow 17^{-}$ \\
994.3 & 31.3 & $0.99(05)$ & $\mathrm{E} 2$ & $17^{-} \rightarrow 15^{-}$ \\
1009.0 & 2.6 & - & & $\rightarrow 20$ \\
1036.3 & 6.4 & $0.62(08)$ & $\mathrm{E} 1$ & $16^{+} \rightarrow 15^{-}$ \\
1039.5 & 2.7 & - & & $6^{+} \rightarrow 4^{+}$ \\
1062.9 & 48.1 & $0.98(04)$ & $\mathrm{E} 2$ & $8^{+} \rightarrow 6^{+}$ \\
1063.8 & 4.0 & - & & $\rightarrow 16^{-}$ \\
1071.6 & 3.3 & - & & $\rightarrow 22^{+}$ \\
1097.8 & 4.8 & $0.99(17)$ & $\mathrm{E} 2$ & $21^{-} \rightarrow 19^{-}$ \\
1118.9 & 14.5 & $0.98(07)$ & $\mathrm{E} 2$ & $6^{+} \rightarrow 4^{+}$ \\
1259.5 & 3.3 & $1.15(31)$ & $\mathrm{E} 2$ & $4^{+} \rightarrow 2^{+}$ \\
1282.0 & 5.0 & $0.54(08)$ & $\mathrm{E} 1$ & $7^{-} \rightarrow 6^{+}$ \\
\hline
\end{tabular}

${ }^{a}$ Energies are estimated to be accurate to $\pm 0.2 \mathrm{keV}$.

${ }^{b}$ Errors on the relative intensities are typically $\leq 5 \%$.

c The DCO results were obtained from a sum of gates on the 658 and $745 \mathrm{keV}$ quadrupole transitions.

${ }^{d}$ Doublet, DCO value given for composite peak. 
Table II. Observed dipole strengths in ${ }^{110} \mathrm{Te}$, assuming $Q_{0}=200$ e.fm ${ }^{2}$. The uncertainties are estimated to be $\leq 10 \%$.

\begin{tabular}{ccccc}
\hline $\begin{array}{c}\mathrm{I} \\
\text { initial }\end{array}$ & $\begin{array}{c}\mathrm{B} 1) / \mathrm{B}(\mathrm{E} 2) \\
\left(\mathrm{fm}^{-2}\right)\end{array}$ & $\begin{array}{c}\mathrm{B}(\mathrm{E} 1) \\
\left(\mathrm{e}^{2} \mathrm{fm}^{2}\right)\end{array}$ & $\begin{array}{c}\mathrm{B}(\mathrm{E} 1) \\
(\text { W.u. })\end{array}$ & $\begin{array}{c}\left|D_{0}\right| \\
(\mathrm{e} . \mathrm{fm})\end{array}$ \\
\hline \hline 18 & $6.3 \times 10^{-7}$ & $0.89 \times 10^{-3}$ & $0.60 \times 10^{-3}$ & 0.087 \\
20 & $9.4 \times 10^{-7}$ & $1.33 \times 10^{-3}$ & $0.91 \times 10^{-3}$ & 0.107 \\
22 & $13.7 \times 10^{-7}$ & $1.95 \times 10^{-3}$ & $1.33 \times 10^{-3}$ & 0.130 \\
\hline
\end{tabular}




\section{FIGURE CAPTIONS}

Fig. 1 - Level scheme for ${ }^{110} \mathrm{Te}$ deduced from this work. Transition energies are given in $\mathrm{keV}$ and relative intensities are represented by the widths of the arrows.

Fig. 2 - Examples of gated coincidence spectra for ${ }^{110} \mathrm{Te}$. Transitions assigned to ${ }^{110} \mathrm{Te}$ are labelled by their energies in $\mathrm{keV}$. Contaminants $\left({ }^{112} \mathrm{Te}\right)$ in $(\mathrm{c})$ are indicated by an asterisk. 


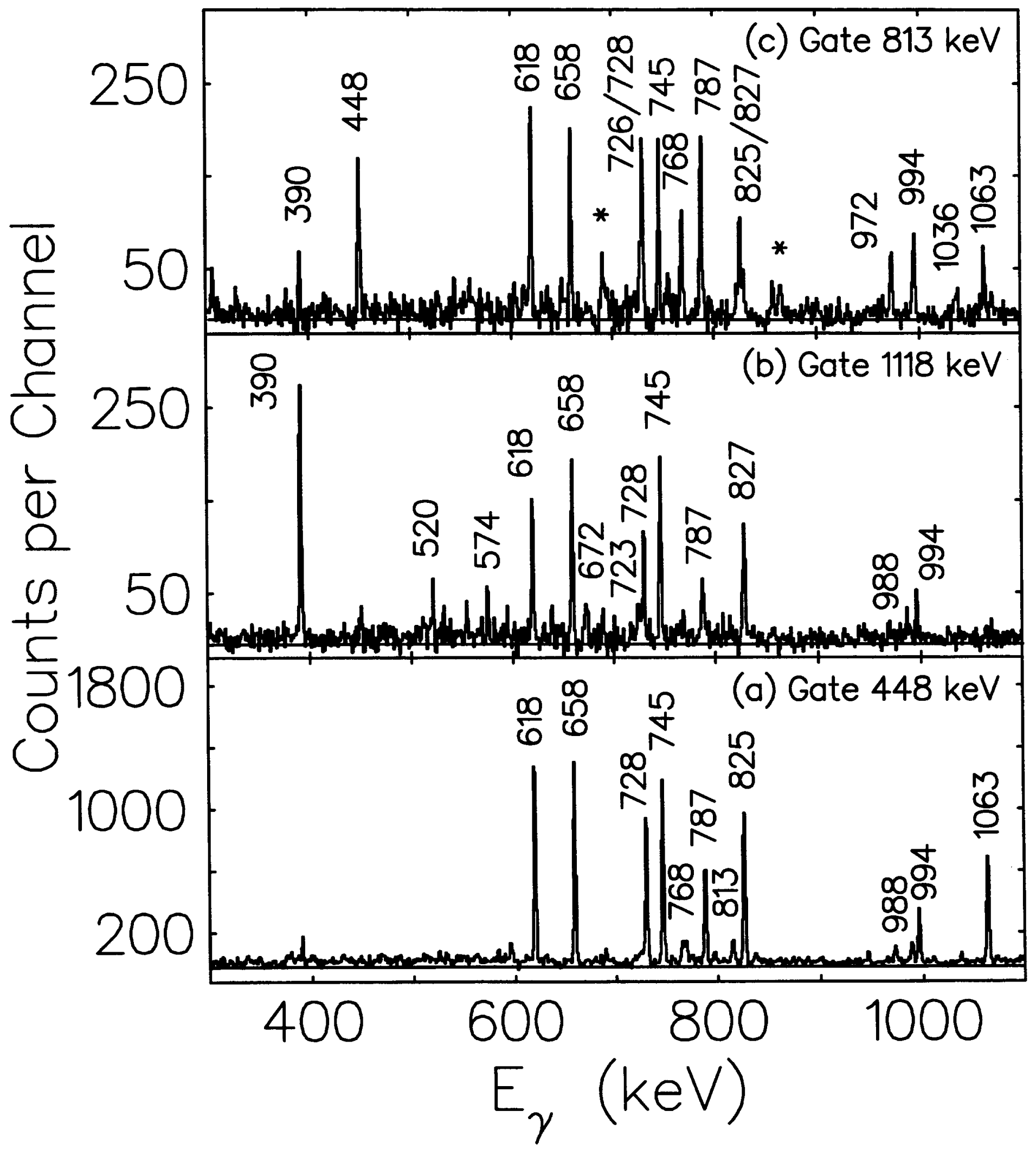




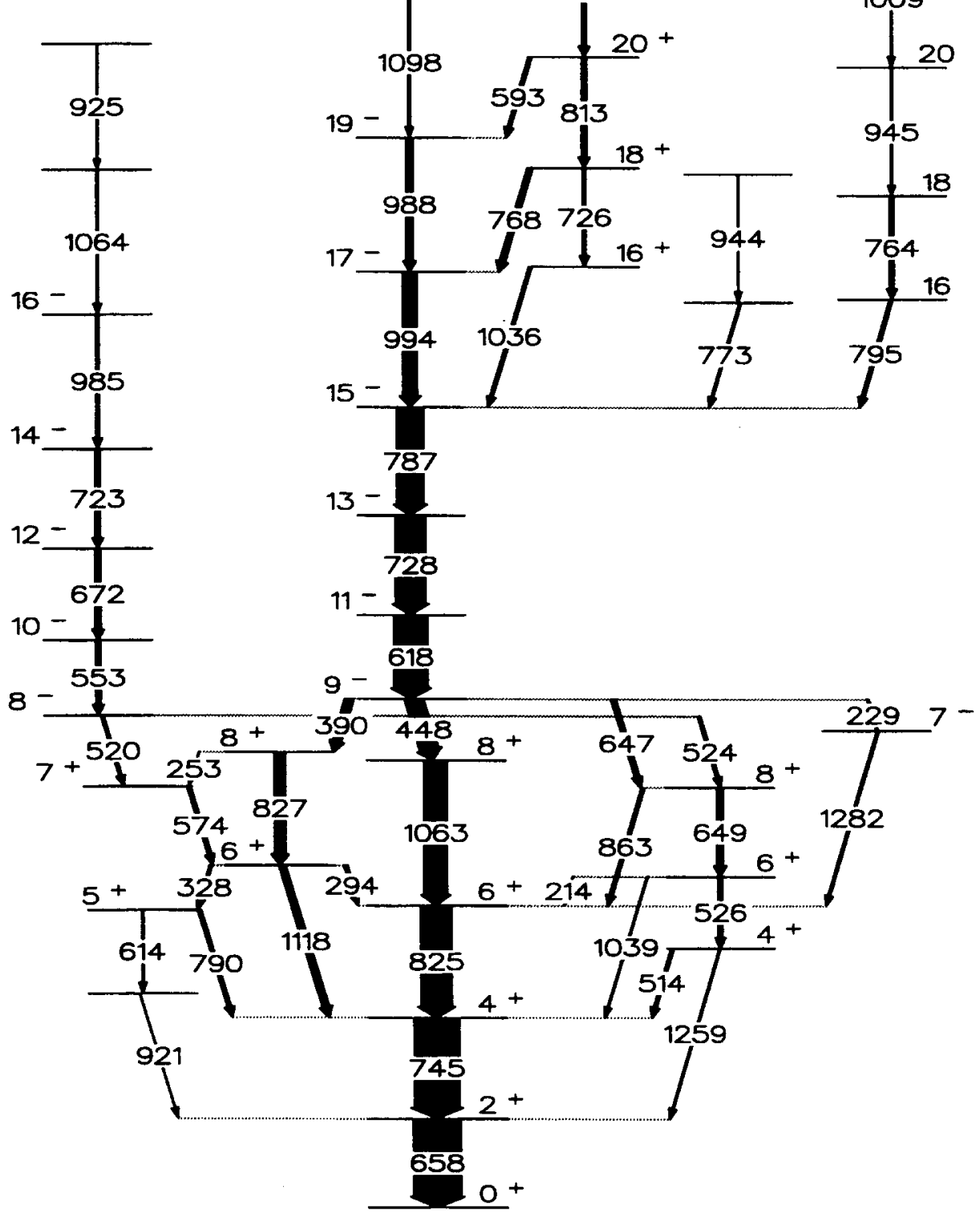

